\title{
Empatía: desde la percepción automática hasta los procesos controlados
}

\section{Empathy: From futomatic Perception to Controlled Processes Empatia: Desde a Percepção futomática até os Processos Controlados}

\author{
Mariana Beatriz López, Vanessa Arán Filippetti, María Cristina Richaud* \\ Centro Interdisciplinario de Investigación en Psicología Matemática y Experimental (CIIPME)
}

Doi: dx.doi.org/10.12804/ap132.1.2014.03

\section{Resumen}

Desde la primera utilización del término empatía en Psicología, a principios del siglo XX, su uso se ha extendido por las distintas ramas de esta ciencia, lo que ha dado lugar a la proliferación de teorías y categorías concebidas para explicar su funcionamiento o describir sus componentes. En el presente artículo se revisan algunas discusiones sobre los alcances y los límites del concepto de empatía y se intenta aclarar las particularidades y las relaciones entre términos que conviven en la investigación sobre el tema: contagio emocional, empatía emocional, toma de perspectiva, teoría de la mente y mentalización. Se propone además una integración de este campo conceptual en un esquema amplio, al buscar dar cuenta de la complejidad del constructo de Empatía. Palabras clave: empatía, empatía emocional, teoría de la mente, mentalización

\section{Abstract}

Since its first use in Psychology at the beginning of the 20th century, the term Empathy has reached the different branches of the aforementioned science, leading to the spread of a number of theories and categories developed in order to explain its functioning or describe its components. In the present paper, some discussions about the scope and the limits of empathy are reviewed in an attempt to clarify the particularities and relations between distinct terms that coexist in the study of this topic: namely, Emotional Contagion, Emotional Empathy, Perspective Taking, Theory of Mind and Mentalizing. Furthermore, we propose an integration of this theoretical field into a broad scheme, in an attempt to account for the complexity of the construct of Empathy.

Key words: empathy, emotional empathy, theory of mind, mentalizing

\section{Resumo}

Desde a primeira utilização do termo Empatia em Psicologia no início do século XX seu uso estendeu-se pelos diferentes ramos desta ciência, dando lugar à proliferação de teorias e categorias concebidas para explicar seu funcionamento ou descrever seus componentes. No presente artigo revisamos algumas discussões sobre os alcances e os limites do conceito de Empatia, tentando esclarecer as particularidades e as relações entre termos que convivem na pesquisa sobre o tema: contágio emocional, empatia emocional, toma de perspectiva,

* $\quad$ Mariana Beatriz López, Centro Interdisciplinario de Investigación en Psicología Matemática y Experimental (CIIPME), Consejo Nacional de Investigaciones Científicas y Técnicas (CONICET); Vanessa Arán Filippetti, CIIPME, CONICET; María Cristina Richaud, CIIPME, CONICET.

La correspondencia relacionada con este artículo debe ser dirigida a Mariana Beatriz López, Centro Interdisciplinario de Investigación en Psicología Matemática y Experimental (CIIPME), Consejo Nacional de Investigaciones Científicas y Técnicas (CONICET), Tte. Gral. Juan D. Perón 2158- Buenos Aires Argentina. Correo electrónico: nanablopez@gmail.com

Para citar este artículo: López, M. B., Filippetti, V. A. \& Richaud, M. C. (2014). Empatía: desde la percepción automática hasta los procesos controlados. Avances en Psicología Latinoamericana, vol. 32(1), pp. 37-51. doi: dx.doi.org/10.12804/apl32.1.2014.03 
teoria da mente e mentalização. Além disso, propomos uma integração deste campo conceitual em um esquema amplo, procurando dar conta da complexidade do constructo de Empatia.

Palavras-chave: Empatia, Empatia Emocional, Teoria da Mente, Mentalização

\section{¿Qué es la empatía?}

Al intentar describir coloquialmente la empatía suele recurrirse a frases como "ponerse en el lugar en los zapatos de los demás", buscando una metáfora para la idea de comprender o sentir lo que el otro siente en determinada situación. Desde el ámbito científico no existe una definición unívoca de empatía. Se trata más bien de un campo conceptual en construcción y discusión, en el que recientemente se han realizado algunos intentos de integración.

Al realizar una primera aproximación, se podría decir que la empatía es la capacidad de comprender los sentimientos y emociones de los demás, basada en el reconocimiento del otro como similar. Es una habilidad indispensable para los seres humanos, teniendo en cuenta que toda nuestra vida transcurre en contextos sociales complejos. Esta naturaleza social hace que el reconocimiento y la comprensión de los estados mentales de los demás, así como la capacidad de compartir esos estados mentales y responder a ellos de modo adecuado, sean tanto o más importantes que la capacidad de comprender y responder adecuadamente a los contextos naturales no sociales.

El término empatía es la traducción del inglés empathy, que a su vez fue traducido del alemán einfühlung por Titchener (1909). El término einfühlung, que significa sentirse dentro de algo o alguien, comenzó a utilizarse en el campo de la Estética alemana de fines del siglo XIX y fue traducido al inglés empathy para ser utilizado en el campo de la psicología experimental de EE.UU en los comienzos del siglo XX (Wispé, 1987). Aunque los psicólogos suelen atribuir a Lipps la primera conceptualización de la empatía, sería más apropiado decir que él fue quien tomó el concepto de la Estética, lo organizó y desarrolló en el campo de la psicología (Wispé, 1987). De acuerdo con esta concepción original, ligada a los desarrollos del autor sobre la experiencia estética (Lipps, 1903), la empatía es la tendencia natural a sentirse dentro de lo que se percibe o imagina, tendencia que permite, en primer lugar, reconocer la existencia de otro (Wispé, 1987). Para Lipps, la imitación, que tiene lugar en distintos niveles, constituye el proceso básico que da lugar a la autoconciencia en la experiencia, y a la conciencia del objeto experienciado. La empatía es, así, la unión entre un sujeto y un objeto artístico, la participación de dos sujetos en la misma experiencia por medio de sus acciones (Morgade Salgado, 2000).

A partir de esta primera utilización del término empatía en psicología de la experiencia estética (Lipps, 1903), su uso se ha ido extendiendo a numerosas ramas de esa ciencia. Desde los teóricos de la personalidad hasta los psicoterapeutas se han servido de él. Se ha empleado también dentro del campo de la psicología del desarrollo, para la comprensión y explicación las conductas altruistas (Wispé, 1987).

Esta expansión del uso del término empatía dentro del campo de la psicología y las neurociencias, ha dado lugar a la proliferación de teorías y categorías para describirlo y explicarlo. Como señalan Preston y de Waal (2002), en la investigación sobre el tema aparecen una serie de términos que comparten aspectos conceptuales y que muchas veces son usados de modo intercambiable. A lo largo de este artículo se intentará clarificar las particularidades y las relaciones entre algunos de ellos: empatía, contagio emocional, toma de perspectiva, teoría de la mente y mentalización, y se presentarán posibles modos de articulación de este campo conceptual.

\section{Diversas aproximaciones al concepto}

A pesar de la considerable atención que el tema ha recibido por parte de los investigadores, no se ha arribado aún a un consenso en cuanto a los procesos básicos o componentes fundamentales de la empatía. Lipps (1903), quien describió el mecanismo de la einfühlung por primera vez dentro del campo de la psicología, se refería a ella como un instinto innato. Para este autor, la percepción de una emoción en otro por medio de su géstica-articula- 
ción de los múltiples gestos individuales-activaba de manera directa esa misma emoción en quien la percibía, sin ninguna intervención de funciones cognitivas al estilo de la toma de perspectiva. A partir de esa primera descripción fueron constituyéndose dos vertientes teóricas: la de los autores que sostuvieron la idea de Lipps de la percepción directa, y la de quienes hicieron mayor hincapié en aspectos cognitivos como la proyección y la imaginación, convirtiendo a la empatía en un sinónimo de la toma de perspectiva y limitándola a aquellos individuos que poseen teoría de la mente (Preston $\&$ de Waal, 2002).

La mayor bondad de los modelos fundados en la percepción directa, que reconocen al contagio emocional y a la imitación como la base de la empatía, reside en que son capaces de dar cuenta de la continuidad del fenómeno entre especies. Los modelos que ponen el acento en el componente cognitivo, por otro lado, subrayan las diferencias entre la empatía humana y fenómenos similares observados en otros animales.

Cada una de estas corrientes teóricas ha encontrado sustento en la moderna investigación en neurociencia. Las teorías que hacen hincapié en la percepción automática de las emociones de los demás han hallado apoyo empírico en las investigaciones sobre neuronas espejo. En esta corriente se encuentra el modelo de Percepción/ Acción, consistente con la Teoría de la Simulación que se describirá a continuación. Por otro lado, las teorías que subrayan los aspectos cognitivos han encontrado apoyo empírico en las investigaciones que muestran la activación temporal y medial de las regiones prefrontales durante la realización de tareas que implican lectura de mente. Este sustrato diferenciado no implica, sin embargo, que ambas perspectivas sean mutuamente excluyentes; por el contrario, es posible su integración por medio de la puntualización de las relaciones entre estos procesos (Rameson \& Lieberman, 2009).

Modelos que hacen hincapié en la percepción directa: E1 Modelo Percepción/Acción y la Teoría de la Simulación. Preston y de Waal (2002) proponen una teoría de la empatía que pone el acento en la percepción directa. Estos autores presentan una explicación de la empatía dividida en dos niveles: la explicación de sus bases últimas y la explicación de sus bases próximas. Las bases últimas refieren al desarrollo filogenético de la empatía. En su descripción, centran la atención en el vínculo emocional innato que caracteriza a los animales que viven en grupo y que en su manifestación básica se presenta como contagio emocional. El contagio emocional se define a partir de la total identificación con el otro, o confusión entre el self y los otros, que conduce a compartir la emoción del otro en un mismo nivel de intensidad. En los seres humanos este proceso puede considerarse el primer nivel de respuesta empática, ya que es la respuesta más básica y es anterior en el desarrollo a cualquier otra.

En cuanto a las bases próximas de la empatía, Preston y de Waal (2002) aluden a su desarrollo en la ontogenia, y basan su exposición en la descripción del mecanismo neurobiológico de base: el Modelo de Percepción/Acción.

El modelo de Percepción/Acción se sostiene en la noción de representaciones compartidas, de acuerdo con el cual el observador experimenta la emoción del observado por compartir con él las representaciones mentales sobre un determinado comportamiento, estado o situación (Rameson \& Lieberman, 2009). La percepción del comportamiento de otro agente activa las representaciones del observador sobre ese mismo comportamiento, lo cual dispara respuestas autonómicas y somáticas que crean una experiencia emocional en el observador que concuerda con la del observado (Preston \& de Waal, 2002). La empatía es vista dentro de este modelo como un proceso automático no consciente (Rameson \& Lieberman, 2009).

Esta concepción se fundamenta a su vez en la continuidad entre acción y cognición basada en los ciclos de percepción/acción. Percepción y acción están entrelazadas funcionalmente desde el nacimiento, de modo que la percepción es un medio para la acción y la acción es un medio para la percepción (Decety \& Jackson, 2004). La actividad espontánea en áreas corticales y subcorticales del cerebro observada en embriones y fetos es la condición base de estos ciclos, cuyo origen anterior al nacimiento asegura en primer lugar la relación 
con el mundo externo (Decety \& Jackson, 2004). Durante el desarrollo postnatal, el gobierno de la actividad motriz se alcanza justamente por medio de la transformación de patrones perceptivos en patrones de coordinación motriz (Sperry, 1952).

La imitación en neonatos es una evidencia conductual de la asociación innata entre percepción y acción (Meltzoff \& Decety, 2003). Esta imitación no constituye solo un mecanismo de resonancia conductual, es decir, una actividad neuronal generada espontáneamente durante la percepción de movimientos, gestos y acciones de otros, porque requiere cierto nivel de identificación con el agente de esas acciones. Hacia los dos meses de edad los niños tienden a imitar acciones humanas pero no los movimientos de objetos, porque de modo implícito comprenden a las personas como similares (Legerstee, 1991). Esto retrotrae a la primera definición que se dio del término empatía, se trata de la capacidad de comprender al otro, basada en el reconocimiento de ese otro como similar. Por lo tanto, la empatía se erige sobre la base misma de la humanidad: la autoconciencia, desarrollada desde el nacimiento, por medio de la interacción con otros, de la conciencia de otros.

La evidencia neurocognitiva de la asociación entre percepción y acción son las neuronas espejo. Numerosas investigaciones han demostrado que gran parte de los circuitos neuronales involucrados en la ejecución de una acción se ponen en funcionamiento también cuando esa acción es observada o imaginada en otro agente (Rizzolatti \& Craighero, 2004). Se ahondará en esos estudios en el apartado correspondiente.

El modelo de Percepción/Acción que presentan Preston y de Waal (2002), como base de la comprensión de la empatía, y Decety y Jackson (2004), como parte de su modelo -que será presentado en un apartado posterior- es consistente con la Teoría de la Simulación (Gallese, 2001). La idea fundamental de la Teoría de la Simulación es que se comprende a los demás al utilizar la propia mente como modelo. Gallese (2001) se basa en la investigación neurocientífica para desarrollar esa teoría, al sostener una afirmación básica compatible con el modelo que se ha expuesto hasta aquí: la capacidad de comprender a otros como agentes con intención no depende exclusivamente de habilidades lingüísticas y mentalísticas, sino que se funda en la naturaleza relacional de la acción (Gallese, 2001).

Cuando se va a realizar una determinada acción se puede prever sus consecuencias. Por medio de un proceso de equivalencia motriz se es capaz de usar esa información para predecir las consecuencias de las acciones de los demás. Se trata de un mecanismo automático, no consciente, de simulación motriz que permite penetrar en el mundo de los demás sin la necesidad de teorizar o de recurrir a un razonamiento proposicional (Gallese, 2001). Para Gallese (2001) la acción es el principio a priori que posibilita los lazos sociales. Por medio del mecanismo de simulación de la acción, cuando se observa a otro individuo actuar, se puede reconocer inmediatamente como agente dirigido a metas, es decir, como similar a nosotros, porque este sustrato neuronal se activa cuando se busca alcanzar esa meta por medio de la propia acción.

A partir de esta concepción, el autor propone ampliar el concepto de empatía para dar cuenta de todos los aspectos de la conducta que permiten establecer una relación significativa con otros individuos, es decir, no limitarlo al ámbito de las emociones y sentimientos (Gallese, 2001).

Gallese (2001) reconoce la base de su concepción en postulados de la fenomenología de Husserl, Stein y Merleau-Ponty, entre otros. De acuerdo con Husserl (1989), lo que hace inteligible la conducta de los demás es el hecho de que su cuerpo se percibe no como un objeto inanimado, sino como algo vivo, análogo al propio cuerpo actuante. Stein (1964) utiliza el concepto de similitud para referirse a esta misma idea, el otro es percibido como un ser similar a nosotros y un componente fundamental de esa similitud reside en la experiencia común de la acción.

\section{La Empatía y las funciones cognitivas superio-} res: Teoría de la Mente, Mentalización y Toma de perspectiva. En las investigaciones que hacen hincapié en los aspectos más cognitivos de la empatía, vinculados a las funciones cognitivas superiores características de los seres humanos, suelen aparecer términos como teoría de la mente, mentalización y toma de perspectiva. Estos términos se utilizan 
muchas veces de modo intercambiable, sin que se proponga una definición que clarifique los alcances y los límites de cada uno.

De acuerdo con Premack y Woodruff (1978), cuando se dice que un individuo tiene teoría de la mente, se hace referencia a que atribuye estados mentales a sí mismo y a otros. Se utiliza el término teoría para referirse a ese sistema de inferencias, en primer lugar, porque los estados mentales atribuidos no son observables directamente -por lo tanto son suposiciones teóricas-y, en segundo lugar, porque este puede ser utilizado para realizar predicciones respecto del comportamiento de otros, es decir, para realizar suposiciones teóricas con respecto a las consecuencias conductuales de los estados mentales atribuidos. Frith y Frith (2006) denominan mentalización al proceso por medio del cual se realizan esas inferencias respecto de los estados mentales propios o ajenos, es decir, al proceso metacognitivo de pensar acerca de los contenidos de la mente de otra persona.

La comprensión de que los otros tienen un mundo mental propio que difiere del nuestro es un paso crítico en el desarrollo de los seres humanos, que generalmente tiene lugar alrededor de los 4 años (Wimmer \& Perner, 1983), y que tiene carácter universal en los seres humanos adultos (Premack \& Woodruff, 1978). Aunque su desarrollo está ligado a la experiencia, no requiere de una pedagogía explícita como las matemáticas o el lenguaje escrito, sino que se adquiere de modo más espontáneo como el caminar o el lenguaje hablado. La enseñanza directa solo puede capacitar para suprimir voluntariamente estas inferencias al describir la conducta de otro (Premack \& Woodruff, 1978).

El ser humano es capaz de inferir distintos tipos de estados mentales, desde la más básica inferencia de intención o propósito hasta la de creencias, pensamientos, conocimiento, supuestos, mentira, confianza, entre otros. Es decir, es capaz de inferir, al utilizar una serie de claves, lo que otra persona cree, piensa, sabe o supone, si finge o si confía en tal o cual cosa, etc. Frith y Frith (2006) hacen referencia a distintos tipos de estados mentales: (a) disposiciones de larga duración -por ejemplo, la persona $\mathrm{X}$ es confiable-; (b) estados emocionales de corta duración -como alegría o enojo-; (c) deseos e intenciones asociadas; (d) creencias sobre el mundo, y (e) intención comunicativa.

Ponemos en marcha la mentalización de modo automático cuando nos encontramos frente a otro a quien consideramos agente, utilizando una serie de claves de diferentes modalidades. Se atiende al rostro, a los movimientos corporales, a la dirección de la mirada, a la entonación o al ritmo de la voz, etc. (Frith \& Frith, 2006). Pero un aspecto fundamental de la mentalización exitosa es la toma de perspectiva, es decir, la capacidad de considerar una situación desde diferentes puntos de vista. Una correcta atribución de creencias se basa en el reconocimiento de que el conocimiento está fundado en la experiencia y, por lo tanto, un individuo que no ha tenido nuestras mismas experiencias puede no saber lo mismo que nosotros (Wimmer, Hogrefe \& Perner, 1988). Esto podría extrapolarse a otros estados mentales, como por ejemplo los deseos y las emociones: una persona que ha tenido experiencias diferentes a nosotros puede sentirse de modo diferente en una misma situación, o puede desear otras cosas en la misma circunstancia.

De hecho, esta habilidad es evaluada generalmente por medio de pruebas en las que los participantes tienen que informar acerca de la falsa creencia de otro individuo, cuyo conocimiento fáctico difiere del que posee el participante, denominadas Pruebas de la Falsa Creencia. La realización con éxito de esas pruebas demuestra la habilidad de una persona de distinguir entre los contenidos de la propia mente y los de la mente del otro, basándose en el conocimiento del que ella dispone (Rameson \& Lieberman, 2009).

Recapitulando, se podría integrar los conceptos desarrollados en esta sección si se considera que la teoría de la mente es la inferencia de estados mentales en otros y en nosotros mismos, que la mentalización es el proceso por medio del cual se realizan esas inferencias y que la toma de perspectiva es un componente de ese proceso, fundamental para que éste sea exitoso.

\section{Algunos intentos de integración}

Más recientemente, algunos autores han propuesto modelos que buscan integrar las distintas 
dimensiones y procesos que se han descrito hasta aquí, atendiendo a la complejidad del constructo empatía. Estos modelos conjugan de distintos modos la experiencia afectiva automática o no consciente del estado emocional observado o inferido, con el reconocimiento y la comprensión de los estados emocionales de los demás por medio de procesos cognitivos controlados, para conformar esquemas comprehensivos más amplios del fenómeno de la empatía.

En su artículo "The Functional Architecture of Human Empathy", Decety y Jackson (2004) presentan un modelo que integra el componente afectivo y el componente cognitivo de la empatía. De acuerdo con estos autores, aunque es posible que el reconocimiento de emociones básicas sea principalmente un proceso directo y automático, que no requiere inferencias ni meta-representaciones, el reconocimiento de emociones más complejas, como las llamadas emociones de autoconciencia, probablemente requiera de un procesamiento cognitivo. Si bien, como se puntualizó anteriormente, las teorías de la empatía basadas en el contagio emocional tienen la virtud de reflejar la continuidad de su desarrollo entre especies (Preston \& de Waal, 2002), para Decety y Jackson (2004) la empatía humana se diferencia de la observada en otras especies por procesos distintivos surgidos en el curso de la evolución, como la toma de perspectiva y la autoconciencia que posibilitan la comprensión de emociones y situaciones más complejas. La empatía humana, a diferencia de la observada en otros animales en general e incluso en otros primates, implica teoría de la mente y mentalización, es decir, la capacidad de predecir la conducta de otros por medio de la atribución de estados mentales independientes (Decety \& Jackson, 2004). Mientras que reflejar la emoción de otro es un proceso básico que puede ocurrir sin intervención de la conciencia, los procesos que caracterizan a la empatía humana, como la toma de perspectiva y la autorregulación, requieren de un procesamiento controlado (Decety \& Jackson, 2004).

El modelo multidimensional que proponen Decety y Jackson (2004) incluye tres componentes que interactúan dinámicamente para producir la empatía humana: (a) emoción compartida entre el observador y el observado, proceso que explican por medio del modelo de Percepción/Acción que ya se ha expuesto; (b) Autoconciencia y Conciencia del otro, que impide la confusión entre el self y el otro a pesar de cierta identificación temporal; $\mathrm{y}$ (c) Flexibilidad Cognitiva para adoptar la perspectiva del otro, que necesita de procesos de regulación.

De acuerdo con estos autores, ninguno de estos tres componentes puede dar cuenta por sí mismo del potencial de la empatía humana y en el funcionamiento normal se encuentran en constante interrelación. Por ejemplo, la emoción compartida sin autoconciencia se convierte en total identificación entre el self y el otro, lo que conocemos como contagio emocional. Por otro lado, cada uno de estos macrocomponentes puede ser analizados en cuanto a sus elementos constitutivos y asociados con un sustrato neurológico particular (Decety \& Jackson, 2004).

En un artículo posterior, Decety y Lamm (2006) completan el desarrollo del modelo presentado hasta aquí al proponer las metacategorías bottom-up y top-down para diferenciar distintos procesos al interior de su modelo. Los procesos bottom-up son los procesos de percepción directa en los que percepción y acción son equivalentes, aquí se incluye el primer componente del modelo. Los procesos top-down son aquellos que implican regulación y control. Estos dos niveles de procesamiento están continuamente interrelacionados. Los procesos bottom-up se activan automáticamente por el input perceptivo y de ellos se deriva la experiencia de compartir emociones con otro -resonancia emocional- basada en el reconocimiento implícito de similitud entre el self y los otros. Las funciones ejecutivas propias de la corteza prefrontal sirven para regular tanto la respuesta emocional como la cognitiva, por medio de la atención selectiva y la autorregulación. Estos procesos superiores se actualizan continuamente en función de la información bottom-up y, a su vez, controlan el nivel inferior por medio de un input top-down. Por lo tanto, la regulación top-down, por medio de las funciones ejecutivas, modula el nivel inferior y le otorga flexibilidad, y hace al individuo menos dependiente de claves externas (Decety \& Lamm, 2006). 
Otro modelo propuesto para integrar las distintas perspectivas analizadas en la investigación futura es el de Rameson y Lieberman (2009). Los autores presentan un esquema que parte de la idea de que es posible adoptar dos modos generales de procesamiento de información respecto del self y de los otros, el procesamiento experiencial y el proposicional. El procesamiento experiencial puede ser pensado como una experiencia fluida, automática y afectiva, mientras que el proposicional es un proceso cognitivo controlado. Para entender a otros, una y otra modalidad de procesamiento se emplean alternativamente en función de diversos factores, entre los que se cuentan los recursos cognitivos, la motivación, las diferencias individuales, la relación entre el observador y el observado y el modo en que la situación se presenta-si se observa directamente, o en una película, o se lee sobre ella en una novela-. Para los autores, ambas son fundamentales para comprender el mundo social por medio de la experiencia de la empatía.

Las diferentes teorías sobre el modo en el que se entiende a los demás y a nosotros mismos podrían ordenarse e integrarse entonces, de acuerdo con estos autores, en función de dos parámetros: el Foco del procesamiento -en el Self o en los otros-y el Modo de Procesamiento -Experiencial o Proposicional-(Rameson \& Lieberman, 2009). El procesamiento experiencial sobre nosotros mismos deriva en las emociones relacionadas con la situación externa, por ejemplo, ante un evento negativo podría derivar en estrés y deseos de evitar la situación displacentera; el procesamiento proposicional sobre nosotros mismos constituye una reflexión metacognitiva sobre los propios pensamientos y emociones, pero ambos exceden el campo de la empatía, que se limita al modo en el que se entiende a otros. Se utiliza un procesamiento experiencial sobre otro, por ejemplo cuando al mirar una película nos metemos de modo automático en los zapatos del personaje y sentimos lo que siente en cada situación, como si lo que a este le ocurre nos estuviera ocurriendo a nosotros mismos. En este modo de procesamiento, se puede incluir lo desarrollado sobre la Teoría de la Simulación. Se utiliza un procesamiento proposicional sobre otro cuando se reflexiona sobre sus pensamientos y sen- timientos, al intentar comprender de acuerdo con la circunstancia por qué se siente como se siente o piensa lo que piensa. Este procesamiento se identifica, por lo tanto, con los aspectos cognitivos de la empatía y es compatible con lo desarrollado sobre la mentalización.

Cada uno de estos modos de procesamiento conlleva una experiencia psicológica particular y puede dar lugar a diferentes comportamientos, desde la comprensión y el altruismo hasta la evitación. Además, de acuerdo con la revisión realizada por Rameson y Lieberman (2009), cada modalidad de procesamiento posee un sustrato neuronal único y a la vez comparte algunos circuitos con los demás.

Por último, cabe mencionar que algunos modelos multidimensionales suman a los aspectos afectivos y cognitivos de la empatía, los vinculados con la conducta. Un ejemplo en esta dirección es la propuesta de Gerdes y Segal (2009), quienes presentan un esquema tridimensional, basándose en los desarrollos de Decety y colaboradores (Decety \& Jackson, 2004; Decety \& Lamm, 2006), pero incorporando la acción empática como parte integrante del modelo. Los tres componentes de su modelo son: (a) La respuesta afectiva a las emociones o acciones de otro; (b) El procesamiento cognitivo de la perspectiva ajena y de la propia respuesta afectiva, y; (c) La toma de decisión consciente para realizar una acción empática. De acuerdo con estos autores, los componentes uno y dos del modelo no pueden dar cuenta cabalmente del fenómeno empático. Ser empático implica experimentar un afecto que se observa o se infiere en otro individuo, procesarlo cognitivamente y realizar una acción voluntaria en consecuencia, es decir, una acción basada en la respuesta afectiva y el procesamiento cognitivo (Gerdes \& Segal, 2009). Desde esta perspectiva, entonces, la conciencia empática de malestar (dolor, angustia) en otros debería derivar en acciones dirigidas a modificar su situación, es decir, debería derivar en la ayuda solidaria o en el altruismo.

En la tabla 1 se propone un ordenamiento de los conceptos y teorías desarrollados en los apartados anteriores, en función de las categorías propuestas por los modelos integrativos que se desarrollan en este apartado. 
Tabla 1

Integración de Modelos Teóricos

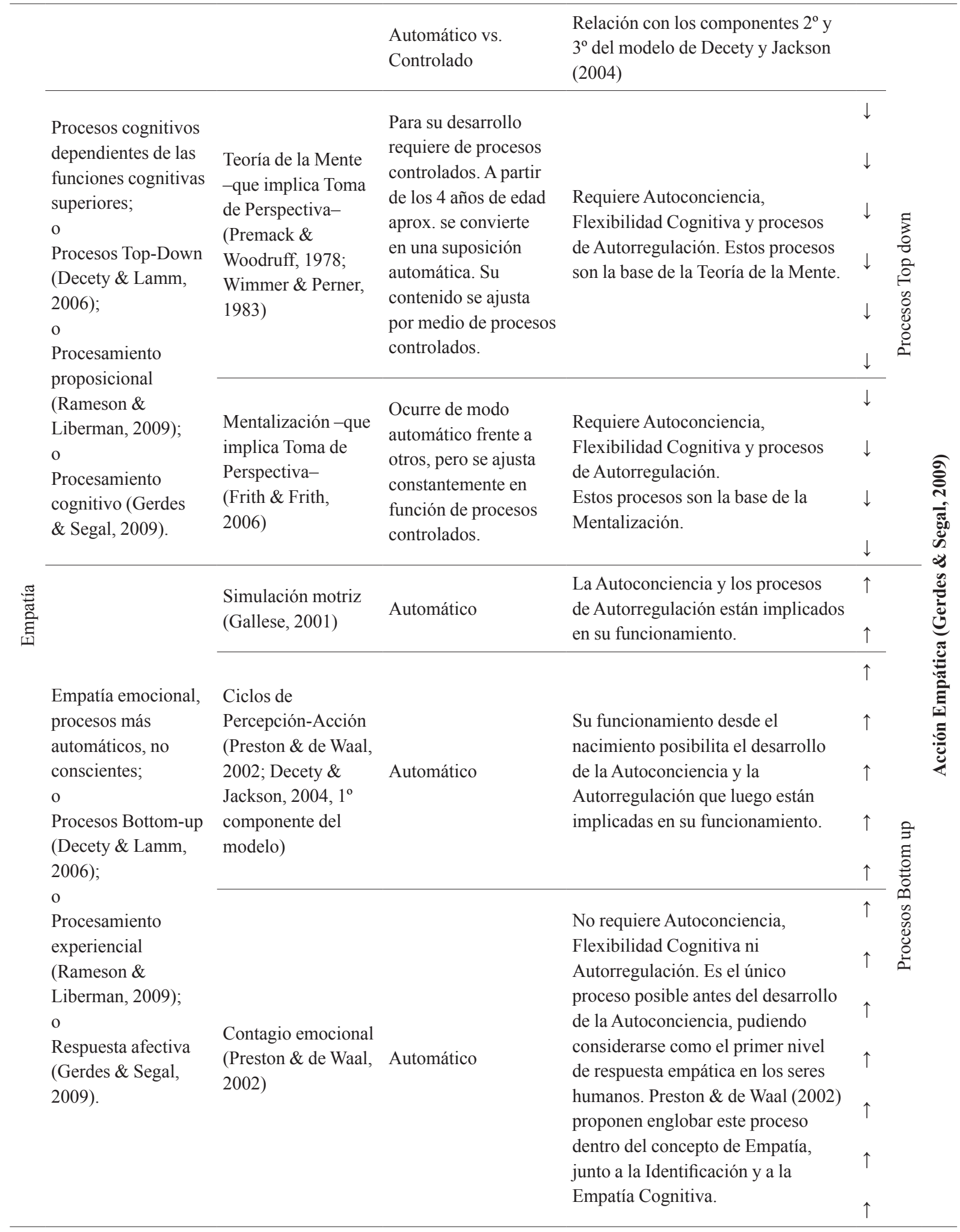

Fuente: elaboración propia. 


\section{Desarrollo de la Empatía: aspectos evolutivos y aprendizaje social}

Existen paralelos entre el desarrollo ontogénico de la empatía en seres humanos y su emergencia filogenética (De Waal, 1996). La inclinación a considerar la empatía humana como un mecanismo complejo que requiere funciones cognitivas superiores, ha tendido a borrar este paralelismo. Sin embargo, es posible considerar las diferencias entre empatía humana y fenómenos similares observados en otros animales como diferencias en el nivel de complejidad y no en la naturaleza del fenómeno en sí (Preston \& de Waal, 2002).

Preston y de Waal (2002) presentan una perspectiva evolucionista y hacen hincapié en lo que la empatía humana tiene en común con la de otros animales sociales y en las presiones evolutivas comunes que han derivado en su desarrollo. Vivir en entornos sociales, característica común de los mamíferos, requiere las habilidades necesarias para comprender y responder a otros de manera adecuada (Brothers, 1989). Según Preston y de Waal (2002) la complejidad social, que está en relación con el tamaño del grupo, constituyó una importante presión evolutiva y, para responder a las demandas que plantea, ha conducido al desarrollo del cerebro hacia la hipertrofiada inteligencia social de los seres humanos.

Estar emocionalmente ligado a otros, es decir, afectado de modo innato por las emociones de los demás, es un requerimiento de la vida en grupo y tiene una serie de ventajas evolutivas (Preston \& de Waal, 2002). En primer lugar, esos lazos constituyen la base que mantiene unida a cualquier sociedad; en segundo lugar, optimizan la seguridad del grupo. Gracias a la vinculación emocional, la alarma de un individuo constituye una alarma para otros. Habiendo muchos ojos dedicados a mantener la seguridad, los individuos pueden dedicar más tiempo a otras actividades.

La vinculación emocional innata con otros facilita también el desarrollo de la relación diádica madre/padre-hijo: cada parte de la díada está conductual y fisiológicamente afectada por la otra, y esta relación contribuye con ambas. El contacto físico y emocional coordinado entre madre/padre- hijo posibilita el desarrollo de las habilidades de regulación emocional del hijo, que determinarán su competencia emocional futura. El llanto o la sonrisa del niño modifican a su vez las respuestas afectivas y emocionales de sus cuidadores, guiando la atención y acción de estos últimos. El contagio emocional del estrés del niño a su cuidador actúa como un estímulo incondicionado y motiva a los cuidadores para actuar antes de que se provoque un evento estresante, genera la necesidad y la motivación para actuar (Preston \& de Waal, 2002).

Estos lazos emocionales son también la base de la empatía y la demanda exitosa de ayuda fuera de la relación diádica madre/padre-hijo (Preston \& de Waal, 2002). Los gritos, el llanto, y otros estímulos similares pueden servir para generar empatía en otros y conseguir ayuda de individuos no familiares. Del mismo modo que al interior de las relaciones familiares básicas, fuera de ellas dichos estímulos son capaces de generar estrés en otros y motivarlos a actuar. Las señales aversivas se desarrollan porque, por su naturaleza, todos desean darles fin. Pero no solo los estímulos aversivos se generalizan más allá del núcleo familiar, sino también aquellos que han servido para obtener contención, afecto y cuidado; esto puede observarse, por ejemplo, cuando dos adultos en una relación de pareja se hablan con voz aniñada (Preston \& de Waal, 2002).

Gracias a las presiones evolutivas pasadas, los cimientos de la empatía están emplazados en nuestro cerebro desde el nacimiento, esperando ser desarrollados por medio de la interacción con otros (Decety \& Jackson, 2004). El impulso hacia el cuidado de las crías, presente en todos los mamíferos, así como la temprana capacidad de los neonatos para distinguir entre el movimiento de otro agente y el de un objeto cualquiera, al dirigir la atención preferentemente hacia el primero (Legerstee, 1991), son probablemente condiciones necesarias para ese desarrollo.

En el apartado sobre el modelo de Percepción/ Acción se habló de otras condiciones que posibilitan la relación con los demás desde el nacimiento. Se presentarán luego los hallazgos respecto a las neuronas espejo, cuyo desarrollo parece también remontarse a etapas muy tempranas. La capacidad 
de un recién nacido de imitar gestos faciales, como la apertura de la boca o la preclusión de la lengua, sugiere que existe cierto mecanismo de espejado desde el nacimiento, al menos en partes del cuerpo que el recién nacido no puede observar directamente y que por lo tanto requieren de un espejado en el cuerpo de otros para el aprendizaje de su control (Gallese, 2001).

Además de un sustrato genético de base, el desarrollo de la empatía requiere entonces de la interacción con otros. La interacción con otros, sustentada en características y recursos innatos, permite la construcción de lazos sociales sin los cuales es improbable que la empatía se desarrolle (Decety \& Jackson, 2004).

En la discusión nature - nurture (naturaleza crianza) respecto a la empatía, se podría dar, por lo tanto, la misma respuesta que en lo referente a muchos otros procesos cognitivos. Es evidente que existen condiciones genéticas que vehiculan la empatía; sus cimientos están en nuestro cerebro (Decety \& Jackson, 2004), pero, como señalan diversos estudios, en su desarrollo interviene la vida social (Barnett, Howard, King \& Dino, 1980; Jenkins Trucker, Updegraff, McHale \& Crouter, 1999; Soenens, Duriez, Vansteenkiste \& Goosens, 2007). Los lazos sociales, originados a partir de una conexión emocional rudimentaria, evolucionan por medio de la experiencia hasta las complejas relaciones humanas adultas.

\section{Las bases neurobiológicas de la Empatía}

Como se señaló anteriormente, la evidencia neurobiológica que sustenta la Teoría Percepción/ Acción (Preston \& de Waal, 2002) y la Teoría de la Simulación (Gallese, 2001) es el sistema de neuronas espejo. Se denomina neuronas espejo a aquellas que se activan tanto cuando se observa a otra persona realizando una acción o experimentando una emoción como cuando se realiza la misma acción o se experimenta la misma emoción (Rizzolatti \& Craighero, 2004). Se trata de una especie de contagio que permite percibir de modo automático el estado de otra persona.

Este sistema de neuronas fue descubierto durante una serie de experimentos de grabación de neurona única-single neuron recording-con monos, en la que los investigadores observaron que un grupo particular de neuronas se activaban tanto durante la ejecución de una actividad manual dirigida a meta -como agarrar, sostener o manipular objetos- como durante la observación de actividades similares realizadas por otros, dándoles entonces a esas neuronas el nombre con que hoy las conocemos (Gallese, Fadiga, Fogassi \& Rizzolatti, 1996; Rizzolatti, Fadiga, Gallese \& Fogassi, 1996).

De acuerdo con Gallese (2001) y su Teoría de la Simulación, la comprensión de una conducta, incluyendo el reconocimiento de la meta a la que va dirigida, requiere de una relación entre el observador y el agente. Esa relación está constituida por la corporalidad de la acción, que implica que observador y agente comparten un esquema motriz de la acción. Es por ello que estas neuronas no se activan durante la observación del movimiento de un agente mecánico. Constituyen un sistema neuronal para el cual la observación y la ejecución de la acción son equivalentes. La observación de la acción produce una activación en la corteza premotora de quien observa, semejante a la que ocurre cuando ese individuo realiza activamente la misma acción (Gallese, 2001).

Posteriormente a las primeras investigaciones con monos, fue demostrada la presencia de un sistema de neuronas espejo similar en los seres humanos, por medio de diferentes técnicas como la simulación magnética transcraneana (Fadiga, Fogassi, Pavesi \& Rizzolatti, 1995) o el electroencefalograma EEG (Cochin, Barthelemy, Lejeune, Roux \& Martineau, 1998). Los estudios con neuroimágenes en seres humanos demuestran que durante la observación de una acción manual se activa un circuito, que se corresponde con el de las neuronas espejo en los monos y que incluye la región del surco temporal superior (STS) izquierdo, el lóbulo parietal inferior y el área de Broca (Rizzolatti et ál., 1996b). Al observar una conducta dirigida a una meta ejecutada con otras partes del cuerpo - por ejemplo, la boca o el pie-se activan sectores diferentes de la corteza premotora, que coinciden con los que se activarían al desarrollar esas acciones específicas (Buccino et ál., 2001). 
Teniendo en cuenta lo desarrollado en el apartado sobre la Percepción/Acción y Teoría de la Simulación, y de acuerdo con la hipótesis de Gallese (2001) que se completa con lo expuesto aquí, acción y percepción son dos caras de la misma moneda porque parte del sistema motriz está implicado en la representación de la acción. La compresión de la acción de otros se alcanza por medio de una simulación, gracias a la equivalencia motriz entre lo que los otros hacen y lo que el observador es capaz de hacer (Gallese, 2001). En otras palabras, la naturaleza relacional de la acción permite establecer una red de significado entre el agente y el observador sin la necesidad de utilizar un razonamiento proposicional.

De acuerdo con otros autores, sin embargo, este sistema no es suficiente para explicar la compleja serie de inferencias que somos capaces de realizar con respecto a los estados mentales de los demás y la consiguiente predicción de su conducta. Para Frith y Frith (2006) las neuronas espejo constituyen solo un primer paso de ese proceso que denominan mentalización. Por ejemplo, el sistema de neuronas espejo está preparado para rastrear continuamente los cambios en los estados emocionales de los demás, pero no es útil por sí solo para comprender actitudes o preferencias estables en los otros (Mitchell, Macrae \& Banaji, 2006) que son, sin embargo, muy importantes para hacer predicciones con respecto a su comportamiento y forman parte de nuestra teoría de la mente.

Además, experimentar la misma emoción que otro no siempre es suficiente para inferir la causa de esa emoción, así como realizar de modo encubierto la misma acción no es siempre suficiente para inferir las metas a las que se dirige o las intenciones detrás de ella (Frith \& Frith, 2006). Si bien, de acuerdo con la Teoría de Simulación se puede utilizar nuestra propia mente como modelo para comprender las causas de las emociones ajenas o los propósitos de las acciones de los demás, esto es válido siempre y cuando los otros sean similares a nosotros, pero no parece suficiente cuando observamos a otro diferente. Mitchell, Macrae y Banaji (2006) observaron que la mentalización frente a alguien que creemos similar -con ideas sociales y políticas similares-involucra una región ventral de la corteza prefrontal medial (mPFC) relacionada con pensamientos autorreferenciales, mientras que la mentalización sobre alguien diferente a nosotros involucra una subregión más dorsal de la mPFC. Si bien la superposición en la activación durante el razonamiento sobre uno mismo y sobre otros similares da apoyo a la Teoría de la Simulación (Mitchell, Macrae \& Banaji, 2006), la investigación demuestra, a su vez, que esa teoría no es suficiente para dar cuenta de las posibilidades de la mentalización en los seres humanos, ya que somos capaces de realizar inferencias sobre los estados mentales y predicciones sobre la conducta de personas muy diferentes, incluso sobre individuos de otras especies.

Frith \& Frith (2006) encuentran en los estudios sobre el correlato neurológico de la mentalización respuestas a las preguntas sobre el modo en que se infieren las causas de las emociones y acciones de los demás y se predice su comportamiento futuro. Los estudios de neuroimagen han demostrado que la corteza prefrontal medial $(\mathrm{mPFC})$ y el giro paracingulado, el STS, los polos temporales, y la encrucijada témporoparietal (TPJ) intervienen durante la ejecución de diferentes pruebas en las que los participantes tienen que pensar sobre los estados mentales de otro agente (Amodio \& Frith, 2006; Frith \& Frith, 2006; Gallagher \& Frith, 2003), lo que también se ha denominado razonamiento proposicional sobre los estados mentales de otros (Rameson \& Lieberman, 2009). Es decir, estas regiones intervienen en pruebas que requieren teoría de la mente.

Durante el proceso de mentalización en la toma de perspectiva, las expresiones faciales pueden ser útiles para inferir las causas de una emoción o una conducta determinada. Por ejemplo, si una persona está asustada será útil analizar qué está mirando y representar el mundo desde su perspectiva visual, ya que allí puede estar la clave para entender su temor. Distintas investigaciones han demostrado que tanto la parte posterior del STS como la TPJ adyacente están involucradas en estos procesos (Frith \& Frith, 2006; Gallagher \& Frith, 2003).

En la comprensión de las situaciones que se observan, se pone además en funcionamiento todo el conocimiento que se ha adquirido acerca del mundo social por medio de las experiencias vitales 
(Frith \& Frith, 2006). Por medio de la experiencia se aprende cuáles son los comportamientos más comunes frente a determinadas situaciones, cuáles son los más correctos o aceptables y cómo suelen comportarse individuos específicos frente a esas situaciones. En el proceso de analizar circunstancias determinadas en función de ese conocimiento adquirido parecen intervenir los polos temporales (Frith \& Frith, 2006; Gallagher \& Frith, 2003). Aparentemente, los polos temporales son zonas de convergencia en las que información simple de diferentes modalidades se cruza para definir en conjunto las características de un individuo o situación (Damasio, Tranel, Grabowski, Adolphs \& Damasio, 2004). La utilización del conocimiento previo no solo permite analizar por qué una persona se siente de una manera o actúa de otra en determinada situación, sino que posibilita la anticipación de sus emociones, conducta o pensamientos, en función del conocimiento de esa persona y esa situación y de las relaciones habituales entre personas y situaciones, es decir, permite hacer predicciones antes que el sistema de neuronas espejo se haya encendido (Frith \& Frith, 2006; Gallagher \& Frith, 2003). El daño en esta zona de la corteza ha sido asociado a la dificultad para utilizar el conocimiento previo en estos análisis (Duval et ál., 2012).

Por último, se ha comprobado en numerosas investigaciones la activación de la mPFC y del giro paracingulado adyacente durante la ejecución de tareas que implican lectura de mente (Amodio $\&$ Frith, 2006; Frith \& Frith, 2006). Se trata de una región bastante amplia en comparación con las analizadas previamente, cuyo rol específico no ha sido aún determinado (Frith \& Frith, 2006). Las personas con lesiones en esta región suelen tener dificultades en distintas pruebas que evalúan teoría de la mente (Stuss, Gallup \& Alexander, 2001).

\section{Discusión}

El concepto de empatía se ha utilizado como marco para incluir un conjunto de procesos que es posible diferenciar desde el punto de vista de su desarrollo, de su localización neuronal y de sus implicancias y consecuencias comportamentales. Algunos de estos procesos son nombrados habi- tualmente con términos como contagio emocional, toma de perspectiva, teoría de la mente y mentalización.

En sus desarrollos teóricos acerca de la empatía, los autores han hecho hincapié alternativamente sobre algunos de ellos. Es particularmente extendida la diferenciación entre quienes han centrado sus explicaciones en los procesos más emocionales, automáticos y no conscientes y quienes se han focalizado en los procesos inferenciales dependientes de las funciones cognitivas superiores, que distinguen a los seres humanos de otros animales. Estos desarrollos, sin embargo, no son necesariamente contradictorios.

A pesar de que es posible individualizar cada uno de los procesos que se han incluido conjunta o alternativamente dentro del concepto de empatía en función de los criterios mencionados, en el funcionamiento normal están interrelacionados. Todos ellos están implicados en la cognición social, es decir, forman parte de los modos en que se percibe a los demás y se piensa sobre ellos. Teniendo esto en cuenta, algunos autores han optado por entenderlos como componentes de la empatía (Decety \& Jackson, 2004), en lugar de analizarlos como mecanismos aislados.

Los intentos de integración buscan incluir desarrollos aparentemente contradictorios en un esquema más amplio que permita comprenderlos como complementarios, como componentes del complejo concepto de empatía. Así, es posible hablar de los aspectos cognitivos y emocionales de la empatía (Decety \& Jackson, 2004), de los procesos Bottom-up y Top-down (Decety \& Lamm, 2006), o del razonamiento proposicional y experiencial (Rameson \& Lieberman, 2009) sin que exista conflicto o inconsistencia al interior de cada par de categorías, aunque nombren procesos susceptibles de ser diferenciados.

Se cree que los esfuerzos en la investigación futura deberían centrarse en completar estos esquemas integradores. La integración requiere, en primer lugar, la clarificación de las particularidades y de los aspectos en común de las distintas categorías y procesos desarrollados en torno al concepto de empatía. En segundo lugar, depende de la posibilidad de elucidar las relaciones de interdependencia 
entre cada uno de los procesos, para convertirlos en componentes de un modelo más global.

Un medio de exploración de la relación entre los componentes de la empatía que no ha sido abordado en este trabajo es el estudio de la psicopatología. Por medio de la utilización de neuroimágenes es posible estudiar las diferencias en las respuestas neuronales de personas en las que la empatía se encuentra comprometida, como por ejemplo personas con autismo o psicopatía, durante tareas diseñadas para evaluar empatía o cualquiera de los procesos implicados en la empatía, profundizando así el reconocimiento de las funciones de las diferentes áreas cerebrales y su interrelación. Por ejemplo, algunas investigaciones indican que los niños con tendencias psicopáticas presentan teoría de la mente y habilidades de mentalización normales, aunque tienen dificultades en el reconocimiento de emociones específicas en otros, como la tristeza y el miedo (Blair, Colledge, Murray \& Mitchell, 2001). Esta evidencia se ha utilizado a menudo para esgrimir que se trata de procesos relativamente independientes, pero lo que está implícito en ella es que el funcionamiento escindido de estos procesos, aunque posible, deriva en un comportamiento socialmente desajustado.

La profundización del estudio de los moderadores de la empatía puede constituir asimismo un medio para alcanzar un conocimiento más cabal de sus componentes. Preston y de Waal (2002) mencionan a la similitud, la familiaridad, la experiencia previa y la notabilidad -salience- como importantes moderadores de la empatía; Rameson y Lieberman (2009) proponen la motivación. La continuidad del estudio de las relaciones entre empatía y personalidad (Bierhoff \& Rohmann, 2004; Del Barrio, Aluja \& García, 2004; Mestre Escrivá \& Samper García, 1997), empatía y género (Mestre Escrivá, Pérez Delgado, Samper García \& Martí Vilar, 1998; Retuerto Pastor, 2004; SchulteRüther, Markowitsch, Shah Fink \& Piefkea, 2008) y, especialmente, entre empatía y apego (Britton \& Fuendeling, 2005; Kestenbaum, Farber, \& Sroufe, 1989; Lenzi, Trentini, Pantano, Macaluso, Lenzi \& Ammaniti, 2012), así como de las relaciones cruzadas entre estas variables (Van der Mark, van
Ijzendoorn \& Bakermans-Kranenburg, 2002), podría resultar también reveladora.

\section{Referencias}

Aluja, A. \& García, L. F. (2004). Relationship between empathy and The Big Five personality traits in a simple of Spanish adolescents. Social Behavior and Personality, 32(7), 677-682.

Barnett, M. A., Howard, J. A., King, L. M. \& Dino, G. A. (1980). Antecedents of Empathy. Retrospective Accounts of Early Socialization. Pers Soc Psychol Bull, 6(3), 361-365. doi: 10.1177/014616728063004

Bierhoff, H-W. \& Rohmann, E. (2004). Altruistic Personality in the Context of the Empathy-Altruism Hypothesis. European Journal of Personality, 18, 351-365. doi: 10.1002/per.523

Blair, J., Colledge, E., Murray, L. \& Mitchell, D. G. (2001). A selective impairment in the processing of sad and fearful expressions in children with psychopathic tendencies. Journal of Abnormal Child Psychology, 29(6), 491-498.

Britton, P. C. \& Fuendeling, J. M. (2005). The relations among varieties of adult attachment and the components of empathy. J Soc Psychol., 145(5), 519-530.

Brothers, L. (1989). A biological perspective on empathy. Am J Psychiatry, 146, 10-19.

Buccino, G., Binkofski, F., Fink, G. R., Fadiga, L., Fogassi, L., Gallese, V., et al. (2001). Action observation activates premotor and parietal areas in a somatotopic manner: An fMRI study. European Journal of Neuroscience, 13, 400-404. doi: 10.1111/j.1460-9568.2001.01385.x

Cochin, S., Barthelemy, C., Lejeune, B., Roux, S. \& Martineau, J. (1998). Perception of motion and qEEG activity in human adults. Electroencephalography and Clinical Neurophysiology, 107(4), 287-295. doi: 10.1016/S0013-4694(98)00071-6

Damasio, H., Tranel, D., Grabowski, T., Adolphs, R. \& Damasio, A. (2004). Neural systems behind word and concept retrieval. Cognition, 92, 179-229. doi: 10.1016/j.cognition.2002.07.001

Decety, J. \& Jackson, P. H. (2004). The Functional Architecture of Human Empathy. Behavioural and 
Cognitive Neuroscience Review, 3(2), 71-100. doi: $10.1177 / 1534582304267187$

Decety, J. \& Lamm, C. (2006). Human Empathy Through the Lens of Social Neuroscience. The Scientific World Journal, 6, 1146-1163. doi: 10.1100/tsw.2006.221

DeWaal, F. B. M. (1996). Good natured: The origins of right and wrong in humans and other animals. Cambridge, MA: Harvard University Press.

Duval, C., Bejanin, A., Piolino, P., Laisney, M., De La Sayette, V., Belliard, S., Eustache, F. \& Desgranges, B. (2012). Theory of mind impairments in patients with semantic dementia. Brain, 135(1), 228-241. doi: 10.1093/brain/awr309

Fadiga, L., Fogassi, L., Pavesi, G. \& Rizzolatti, G. (1995). Motor facilitation during action observation: A magnetic stimulation study. Journal of Neurophysioogy., 73, 2608-2611.

Frith, C. D. \& Frith U. (2006). The Neural Basis of Mentalizing. Neuron, 50(4), 531-534. doi: 10.1016/j. neuron.2006.05.001

Gallagher, H. L. \& Frith, C. D. (2003). Functional imaging of 'theory of mind'. Trends in Cognitive Sciences, 7(2), 77-83. doi:10.1016/S13646613(02)00025-6

Gallese, V., Fadiga, L., Fogassi, L. \& Rizzolatti, G. (1996). Action recognition in the premotor cortex. Brain, 119(2), 593-609. doi: 10.1093/brain $/ 119.2 .593$

Gallese, V. (2001). The 'Shared Manifold' Hypothesis. From Mirror Neurons To Empathy. Journal of Consciousness Studies, 8(5-7), 33-50.

Gerdes, K. E. \& Segal, E. A. (2009). A Social Work Model of Empathy. Advances in Social Work, 10(2), 114-127.

Jenkins Tucker, C., Updegraff, K. A., McHale, S. M. \& Crouter, A. C. (1999). Older Siblings as Socializers of Younger Siblings' Empathy. The Journal of Early Adolescence, 19(2), 176-198. doi: $10.1177 / 0272431699019002003$

Kestenbaum, R., Farber, E. A. \& Sroufe, L. A. (1989). Individual differences in empathy among preschoolers: Relation to attachment history. New Directions for Child and Adolescent Development, 1989(44), 51-64.

Legerstee, M. (1991). The role of person and object in eliciting early imitation. Journal of Expe- rimental Child Psychology, 51, 423-433. doi: 10.1016/0022-0965(91)90086-8

Lenzi, D., Trentini, C., Pantano, P., Macaluso, E., Lenzi, G. L. \& Ammaniti, M. (2012). Attachment models affect brain responses in areas related to emotions and empathy in nulliparous women. Hum Brain Mapp. [Epub ahead of print]. doi: 10.1002/ hbm. 21520 .

Lipps, T. (1903). Einfühlung, innere Nachahmung und Organempfindung. Archiv für die Gesamte Psychologie, 1, 465-519.

Meltzoff, A. N. \& Decety, J. (2003). What imitation tells us about social cognition: a rapprochement between developmental psychology and cognitive neuroscience. Biological Sciences, 358, 491-500. doi: 10.1098/rstb.2002.1261

Mestre Escrivá, V. \& Samper García, P. (1997). Empatía en la teoría de la personalidad: G. Allport y los estudios actuales sobre el tema. Revista de Historia de la Psicología, 18(1-2), 191-203.

Mestre Escrivá, V., Delgado, E. P., Samper García, P. \& Martí Vilar, M. (1998). Diferencias de género en la empatía y su relación con el pensamiento moral y el altruismo. Anales de la Revista de Psicología General y Aplicada, 3.1.1. Recuperado de http:// fs-morente.filol.ucm.es/Publicacioes/Ibersicologia/mestre $1 /$ mestre $1 . h t m$

Mitchell, J. P., Macrae, C. N. \& Banaji, M. R. (2006). Dissociable Medial Prefrontal Contributions to Judgments of Similar and Dissimilar Others. Neuron, 50, 655-663. doi: 10.1016/j.neuron.2006.03.040

Morgade Salgado, M. (2000). Del valor estético de la empatía al negocio inteligente de las emociones. La psicología estética de Theodor Lipps a las puertas del tercer milenio. Revista de historia de la psicología, 21(2-3), 359-372.

Premack, D. \& Woodruff, G. (1978). Does the chimpanzee have a theory of mind? The Behavioral \& Brain Sciences, 4, 515-526.

Preston, S. D. \& de Waal, F. B. M. (2002). Empathy: Its ultimate and proximate bases. Behavioral \& Brain Sciences, 25(1), 1-20. doi:10.1017/ S0140525X02000018

Rameson, L. T. \& Lieberman, M. D. (2009). Empathy: A social Cognitive Neuroscience Approach. Social and Personality Psychology Compass, 3(1), 94-110. doi. 10.1111/j.1751-9004.2008.00154.x 
Retuerto Pastor, A. (2004). Diferencias en empatía en función de las variables género y edad. Apuntes de Psicología, 22(3), 323-339.

Rizzolatti, G. \& Craighero, L. (2004). The mirrorneuron system. Annual Review of Neuroscience, 27, 169-192. doi: 10.1146/annurev.neuro.27.070203.144230

Rizzolatti, G., Fadiga, L., Gallese, V. \& Fogassi, L. (1996a). Premotor cortex and the recognition of motor actions. Cognitive Brain Research, 3(2), 131-141. doi:10.1016/0926-6410(95)00038-0

Rizzolatti, G., Fadiga, L., Matelli, M., Bettinardi, V., Paulesu, E., Perani, D., et al. (1996b), Localization of grasp representations in humans by PET: 1. Observation versus execution. Experimental Brain Research, 111, 246-252. doi: 10.1007/ BF00227301

Schulte-Rüther, M., Markowitsch, H. J., Shah, N. J., Fink, G. R. \& Piefkea, M. (2008). Gender differences in brain networks supporting empathy. NeuroImage, 42, 393-403.

Soenens, B., Duriez, B., Vansteenkiste, M. \& Goosens, L. (2007). The Intergenerational Transmission of Empathy-Related Responding in Adolescence: The Role of Maternal Support. Pers Soc Psychol Bull, 33(3), 299-311. doi: 10.1177/0146167206296300
Sperry, R. W. (1952). Neurology and the mind-body problem. American Scientist, 40(2), 291-312.

Stuss, D. T., Gallup, G. G. Jr. \& Alexander, M. P. (2001). The frontal lobes are necessary for 'theory of mind'. Brain, 124(2), 279-286. doi: 10.1093/brain $/ 124.2 .279$

Titchener, E. (1909). Experimental psychology of the thought processes. New York: Macmillan.

Van der Mark, I. L., Van IJzendoorn, M. H. \& Bakermans-Kranenburg, M. J. (2002). Development of Empathy in Girls During the Second Year of Life: Associations with Parenting, Attachment, and Temperament. Social Development, 11(4), 451-468.

Wimmer, H. \& Perner, J. (1983). Beliefs about beliefs: Representation and constraining function of wrong beliefs in young children's understanding of deception. Cognition, 13(1), 103-128. doi: 10.1016/0010-0277(83)90004-5

Wimmer, H., Hogrefe, G. J. \& Perner, J. (1988). Children's understanding of informational access as source of knowledge. Child Development, 59(2), 386-396.

Wispé, L. (1987). History of the concept of empathy. En Eisenberg, N. \& Strayer J. (Ed.) Empathy and its development, New York: Cambridge University Press.

\section{Fecha de recepción: 18 de agosto de 2012 Fecha de aceptación: 1 de marzo de 2013}


